\title{
Noninvasive Measurement of the Pressure Distribution in a Deformable Micro-Channel
}

\author{
O. Ozsun, V. Yakhot, and K. L. Ekinc: \\ Mechanical Engineering Department and the Photonics Center, Boston University, Boston, Massachusetts 02215, USA
}

\begin{abstract}
Direct and noninvasive measurement of the pressure distribution in test sections of a microchannel is a challenging, if not an impossible, task. Here, we present an analytical method for extracting the pressure distribution in a deformable micro-channel under flow. Our method is based on a measurement of the channel deflection profile as a function of applied hydrostatic pressure; this initial measurement generates "constitutive curves" for the deformable channel. The deflection profile under flow is then matched to the constitutive curves, providing the hydrodynamic pressure distribution. The method is validated by measurements on planar micro-fluidic channels against analytic and numerical models. The accuracy here is independent of the nature of the wall deformations and is not degraded even in the limit of large deflections, $\zeta_{\max } / 2 h_{0}=\mathcal{O}(1)$, with $\zeta_{\max }$ and $2 h_{0}$ being the maximum deflection and the unperturbed height of the channel, respectively. We discuss possible applications of the method in characterizing micro-flows, including those in biological systems.
\end{abstract}

\section{INTRODUCTION}

Since the early experiments of Poiseuille [1] more than two centuries ago, the craft of measuring flow fields in tubes and pipes has been perfected. Even so, the resolution limits of these exquisite experimental probes - such as the pitot tube [2] or the hot wire anemometer [3] - are quickly being approached, given recent advances in micron and nanometer-scale technologies. One frequently encounters micro- [4, 5] and nano-flows [6], which come with smaller length scales [7] and shorter time scales [8, 9] than can be resolved by the commonly available probes. For instance, in a pressure-driven micro-flow, one must insert micron-scale pressure transducers in test sections in order to determine the local pressure drops [10 15]. As the size of a probe becomes comparable to or even bigger than the flow scale itself, measurement of the distribution of flow fields becomes problematic.

Although the tools of macroscopic fluid mechanics may not easily be scaled down, the materials and techniques of micro-fluidics offer unique measurement approaches. Most micro-channels in lab-on-chip systems, for instance, are made up of flexible materials [16, 17]. This provides the possibility of probing a flow by monitoring the response of the confining micro-channel to the flow. In other words, the local (position-dependent) deflection $\zeta$ of the deformable walls of a micro-channel may enable the accurate determination of the pressure field (or the velocity field) under flow. The challenge in this approach, of course, is characterizing the interactions between a deformable body and a flow [18 20]. This is not a simple task, especially in the limit of large deflections. To accurately predict a flow bounded by a deformable wall, one needs to determine the hydrodynamic fields as well as the wall deformations consistently. This requires solving coupled fluid-structure equations [21, 22], often in situa-

\footnotetext{
*e-mail ekinci@bu.edu
}

tions where constitutive relations or parameters describing fluid-structure interactions are not available. Even if these relations and parameters are known, numerical approaches are often expensive.

To make the above-discussion more concrete, let us consider a steady pressure-driven flow between an infinite rigid plate at $y=0$ and a deformable wall at $y=2 h_{0}+\zeta(x)$, where $\zeta(x)$ is the local deflection of the deformable wall due to the local pressure $p(x)$ as shown in figure $1(\mathrm{a})$. The equations for incompressible steady flow $\left(\partial_{x} u+\partial_{y} v=0\right)$,

$$
\begin{gathered}
u \partial_{x} u+v \partial_{y} u=-\partial_{x} p+\nu\left(\partial_{x}{ }^{2}+\partial_{y}{ }^{2}\right) u, \\
u \partial_{x} v+v \partial_{y} v=-\partial_{y} p+\nu\left(\partial_{x}{ }^{2}+\partial_{y}{ }^{2}\right) v,
\end{gathered}
$$

are to be solved subject to boundary conditions $\left.u\right|_{B}=$ $\left.v\right|_{B}=0$. All the variables in (1) have their usual meanings [see figure $1(\mathrm{a})$ ], with $\nu$ being the kinematic viscosity. In general, solution to these equations, accounting for inlet and outlet effects are impossibly difficult. However, if the channel is long such that $\frac{2 h_{0}+\zeta_{\max }}{l} \ll 1$, where $\zeta_{\max }$ is the maximum deflection and $l$ is the length of the channel, we can write the local solution for the average velocity $\bar{u}(x)$ as

$$
\bar{u}(x) \approx \frac{1}{12 \eta}\left[2 h_{0}+\zeta(p(x))\right]^{2} \partial_{x} p .
$$

Here, $\eta$ is the dynamic viscosity, and $\zeta(x)=\zeta(p(x))$ is a local constitutive relation, which determines the dependence of the wall deflection $\zeta(x)$ on $p(x)$. No particular form for this dependence (e.g., elastic) is assumed a priori. In order to find the flow rate and the wall stresses, we need accurate information on $p(x), \zeta(x)$, and the constitutive relation $\zeta(x)=\zeta(p(x))$. If the flow rate is given, the problem becomes somewhat simplified, but still remains rather complex to be handled numerically or analytically.

In this manuscript, we describe a method to close (1) in a deformable channel using independent static measurements of $\zeta=\zeta(p)$. Using this method, we extract 
the pressure distribution in a planar channel flow and validate our measurements against the analytic approximation in (2) for a long channel. Our method does not depend upon the particulars of the local constitutive relation $\zeta(x)=\zeta(p(x))$. In other words, it remains independent of the nature of the wall response, providing accurate results for buckled walls and elastically stretching walls alike.

\section{EXPERIMENTAL SYSTEM}

To test these ideas, we have fabricated planar microchannels with deformable walls and measured the deformations of these micro-channels using optical techniques under different conditions - following the work of Gervais et al. [16]. Figure 1(b) is a rendering of one of our micro-channels under pressure. The inset shows how the channel is formed: a rigid bottom wall and a deformable top wall are held together by clamps, and the channel is sealed by a gasket. The in-plane linear dimensions of the channels are $l \times w=15.5 \times 1.7 \mathrm{~mm}^{2}$. The distance $2 h_{0}$ between the undeflected top wall and the rigid bottom wall is set by a precision metal shim (in the range $100 \mu \mathrm{m} \lesssim 2 h_{0} \leq 250 \mu \mathrm{m}$ ); optical interferometry is employed to independently confirm the $2 h_{0}$ values. Different materials with varying thicknesses $t$ are used to make the deformable walls. In three of the channels studied here, the deformable walls are ultrathin silicon nitride (SiN) membranes fabricated on a thick silicon handle chip $(t \approx 500 \mu \mathrm{m})$. In the other channels, the compliant walls are made up of thicker elastomer (PDMS) layers. Various parameters of all the micro-channels used in this study are given in table 【.

After the micro-channels are formed, they are connected to a standard microfluidic circuit equipped with pressure gauges. In the hydrostatic measurements, the outlet of the micro-channel is clogged, and a water column is used to apply the desired pressure. In flow measurements, a syringe pump is inserted into the circuit to provide the flow.

\section{RESULTS AND DISCUSSION}

\section{A. Hydrostatic Loading}

First, the channels are characterized under hydrostatic loading. In these experiments the inlet port is connected to a water column with the outlet clogged, and hydrostatic pressure $p$ is applied on the channel by raising the water column. The resulting position-dependent deflection field $\zeta_{2 d}(x, z)$ of the compliant wall is measured using white light interferometry [23] at each pressure. In figure $1(\mathrm{~b}), \zeta_{2 d}(x, z)$ of the deformable top wall of sample S1 $\left(t=200 \mathrm{~nm}\right.$ and $2 h_{0}=175 \mu \mathrm{m}$; table \ first row $)$ at $p=2.3 \mathrm{kPa}$ is shown. Cross-sections along the $x$ and $z$ axes taken from this profile are shown in figure 1(c). Because the cross-sections are parabolic in the $z$-direction, we define an average or one-dimensional wall deflection $\zeta(x)$ as

$$
\zeta(x)=\frac{1}{w} \int_{-w / 2}^{+w / 2} \zeta_{2 d}(x, z) d z \approx \frac{2}{3} \zeta_{2 d}(x, z=0) .
$$

Here, $\zeta_{2 d}(x, z=0)$ is the maximum value of the parabolic cross-section, and the factor $\frac{2}{3}$ comes from the integration. Similarly defined $\zeta(x)$ will allow us to perform a one-dimensional analysis in the hydrodynamic case. In figure 1(d), we plot $\zeta(x)$ for the same channel at several different hydrostatic pressures, $100 \mathrm{~Pa} \leq p \leq 50$ $\mathrm{kPa}$. These are the position-dependent (local) constitutive curves. Because of the clamping stresses, the deformable wall is initially in a buckled state. At low $p$, the wall deformation remains in the negative $y$ direction. As $p$ is increased, the wall response becomes elastic, and the wall stretches like a membrane. Also, a small asymmetry is noticeable in $\zeta(x)$, caused by the deformation of the silicon chip during clamping. Figure $1(\mathrm{e})$ and (f) shows the peak deflection $\zeta_{p}$, which typically occurs at $(x, z) \approx\left(\frac{l}{2}, 0\right)$, as a function of $p$ for the elastomer (PDMS) and SiN walls, respectively. Each deformable wall in figure 1(e) and (f) has a constitutive $\zeta_{p}$ vs. $p$ curve, determining the behavior of the entire wall. The thin nitride walls shown in figure $1(\mathrm{f})$ obey the wellknown elastic shell model at high $p, \zeta_{p} \sim p^{1 / 3}[24]$. The elastomer walls in figure 1(e) follow a different power law from the SiN ones, presumably because they are much thicker and bending dominates their deformation. There is no noticeable universality in the $\zeta_{p}$ vs. $p$ data - i.e., the nature of the wall response and thus the constitutive relations are material and geometry (thickness) dependent. Our flow results below, however, remain independent of the wall response.

\section{B. Flow Measurements}

Next, we perform flow measurements in each microchannel. The results from all five channels are shown in figure 2(a). In the experiments, we establish a constant volumetric flow rate $Q$ through each channel using a syringe pump and measure the pressure drop between the inlet and outlet using a macroscopic transducer. We prefer to plot $Q$ as the independent variable because the experiments are performed by varying $Q$ and measuring the pressure drop. In all measurements, a small pressure drop occurs in the rigid inlet and outlet regions of the channel. This is because of the finite size of the connections to the macroscopic pressure transducers. Knowing the geometry of the rigid regions, we determine the pressure drop in these regions from flow simulations (see Appendix A for details). Subsequently, we subtract this "parasitic pressure drop" from the measured pressure drop. In sum- 
(a)

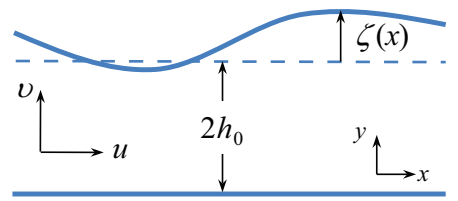

(d)
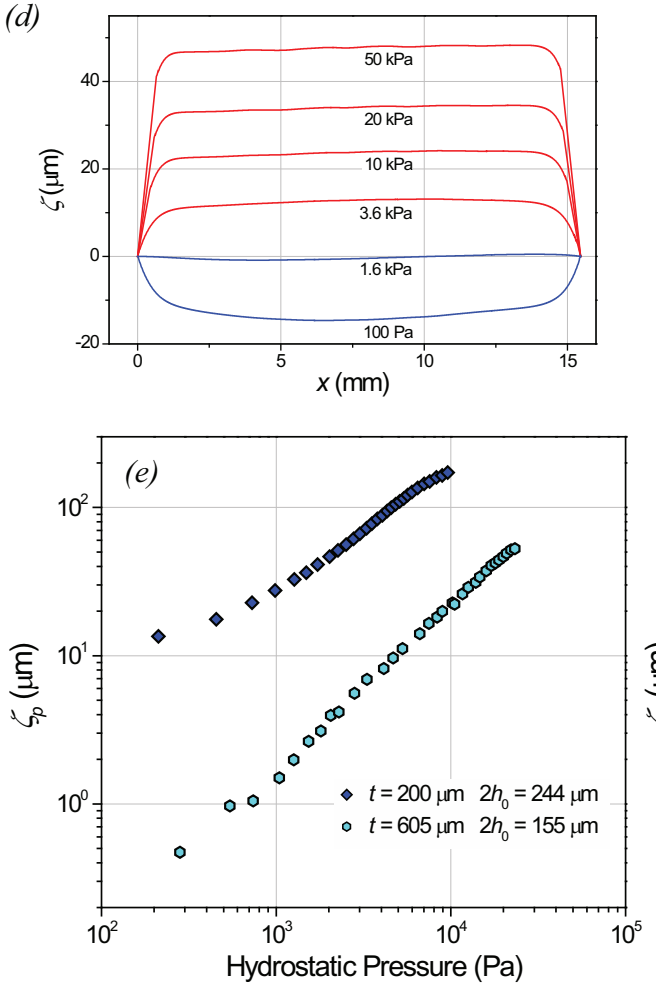

(b)
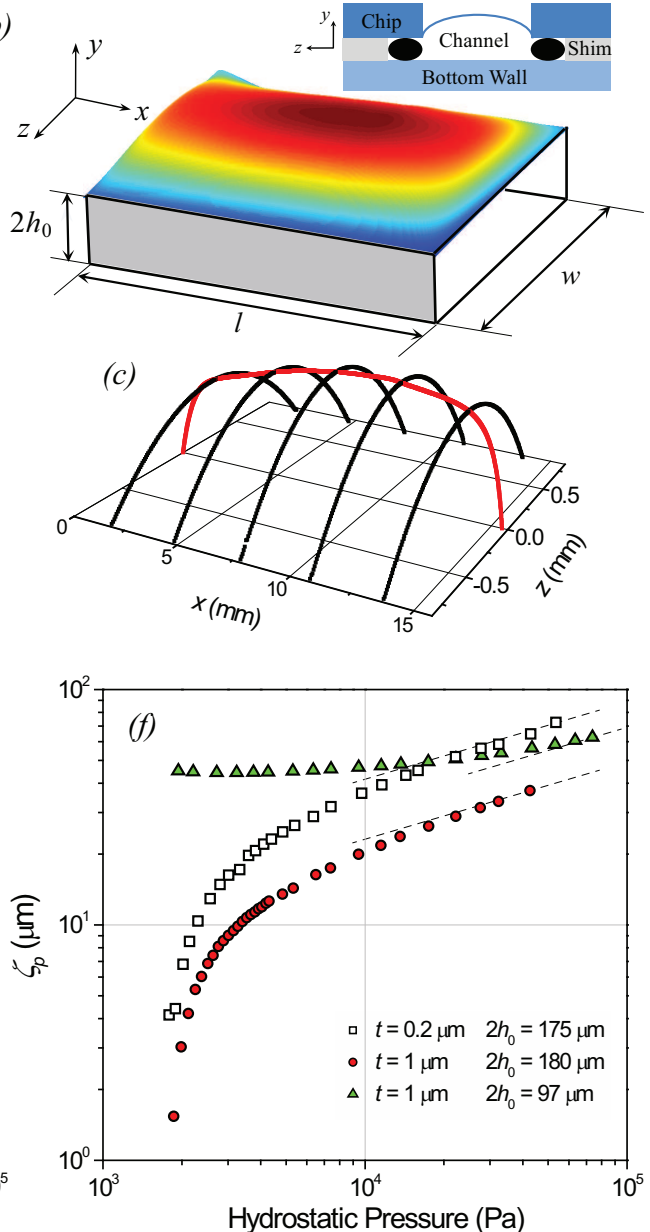

FIG. 1. (a) A one-dimensional channel with a deformable top wall. (b) The two-dimensional deflection $\zeta_{2 d}(x, z)$ of $\mathrm{S} 1(t=200$ $\left.\mathrm{nm}, 2 h_{0}=175 \mu \mathrm{m}\right)$ under hydrostatic pressure of $p=2.3 \mathrm{kPa}$ as measured optically. The inset shows a cross-sectional view of the channel. Clamps hold the chip (with the thin membrane at the center) and the bottom wall together. The two walls are separated by a precision shim; an O-ring (black ovals) seals the channel. (c) Cross-sectional line scans from the image in (b) showing the parabolic $z$-profile of the deformable wall under pressure. (d) The one-dimensional (average) wall deflection $\zeta(x)$ at several different pressures for the same sample. The indicated values are gauge pressure values. These are the local constitutive curves. (e) The peak deflection $\zeta_{p}$ of the PDMS deformable walls as a function of pressure $p$. (f) $\zeta_{p}$ of the SiN deformable walls as a function of $p$; the dashed lines are the $p^{1 / 3}$ asymptotes. Note that the $\zeta_{p}$ and the $p$ axes do not cover the same ranges in (e) and (f). Error bars are smaller than the symbols.

mary, $\Delta p_{t}$ in the plots in figure 2 (a) corresponds to the corrected pressure drop in the compliant section of the channel as measured by a macroscopic transducer (hence, the subscript " $t$ "). Figure 2(b) shows the channel deflection $\zeta(x)$ at several different flow rates for $\mathrm{S} 1(t=200$ $\mathrm{nm}$ and $\left.2 h_{0}=175 \mu \mathrm{m}\right)$. Returning to table $\llbracket$, we now clarify that $\zeta_{\max }$ corresponds to the maximum deflection of the channel at the highest applied flow rate.

\section{Simple Fits}

Before we present our method for analyzing the flow, we attempt to fit the experimental $\Delta p_{t}$ vs. $Q$ data to the theory described above in Eqs. (1/2). Because our channels have a finite width $w$, the result in (2) must be modified slightly. The simplest approach is to use a linear approximation for the local pressure drop based on the hydraulic resistance per unit length, $r(x)$, of the channel. In a long channel at low Reynolds number, $\partial_{x} p \approx \operatorname{Qr}(x)$. The total pressure drop between the inlet and outlet can then be found as $\approx Q \int_{0}^{l} r(x) d x$ [25]. In our analysis, we approximate our channel as a channel of rectangular cross-section of $w \times 2 h(x)$, where $2 h(x)=2 h_{0}+\zeta(x)$. Then [26],

$$
r(x) \approx\left(\frac{1}{1-0.63\left(\frac{2 h(x)}{w}\right)}\right) \frac{12 \eta}{w[2 h(x)]^{3}}
$$




\begin{tabular}{|c|c|c|c|c|c|c|}
\hline Sample & Material & $\begin{array}{l}\text { Wall thickness } \\
\qquad t(\mu \mathrm{m})\end{array}$ & $\begin{array}{c}\text { Unperturbed height } \\
2 h_{0}(\mu \mathrm{m})\end{array}$ & $\mathrm{Re}$ & $\begin{array}{l}\text { Max. defl. Max. } \\
\zeta_{\max }(\mu \mathrm{m}) Q_{\max }\end{array}$ & $\begin{array}{l}\text { flow rate } \\
(\mathrm{ml} / \mathrm{min})\end{array}$ \\
\hline$\overline{\mathrm{S} 1}$ & $\overline{\mathrm{SiN}}$ & 0.2 & 175 & $70-1200$ & 33 & 70 \\
\hline $\mathrm{S} 2$ & $\mathrm{SiN}$ & 1 & 180 & $100-1200$ & 20 & 70 \\
\hline S3 & $\mathrm{SiN}$ & 1 & 97 & $250-1300$ & 37 & 70 \\
\hline $\mathrm{S} 4$ & PDMS & 200 & 244 & $200-800$ & 86 & 50 \\
\hline S5 & PDMS & 605 & 155 & $200-900$ & 25 & 50 \\
\hline
\end{tabular}

TABLE I. Parameters of the channels (first four columns), the range of Reynolds numbers (Re), and the maximum channel deflection $\zeta_{\max }$ attained under the maximum flow rate $Q_{\max }$. The Re is found by averaging $\operatorname{Re}_{x}=\frac{2 Q}{\nu[w+2 h(x)]}$ over the channel.

With the $2 h(x)$ data available from optical measurements, we calculate $r(x)$ and integrate it along the length of the channel for all flow rates to find the pressure drop. The calculated $\Delta p_{t}$ are shown in figure 2 as dashed lines. It is difficult to determine the source of the disagreement between the data and the fits in some cases. The flow in the inlet and outlet regions may still be contributing to the error - even after subtraction. Another source of error may be the boundary between the compliant and rigid regions of the channel.

\section{Measurement of the Pressure Distribution}

We now turn to the main point of this manuscript. Our method is illustrated in figure 3 . The curves in the background in figure 3(a) are the now-familiar $\zeta(x)$ curves of $\mathrm{S} 1\left(t=200 \mathrm{~nm}\right.$ and $\left.2 h_{0}=175 \mu \mathrm{m}\right)$ under different $h y$ drostatic pressures [cf. figure 1(d)]. These serve as the constitutive curves. On top of these hydrostatic profiles, we overlay two different hydrodynamic profiles (thicker lines) at flow rates of $Q=5 \mathrm{ml} / \mathrm{min}$ and $Q=70 \mathrm{ml} / \mathrm{min}$. The assumption here is that, under equilibrium, $\zeta(x)$ only depends upon $p(x)$, providing us with the constitutive relation $\zeta(x)=\zeta(p(x))$. We determine the positions where the dynamic profiles intersect with the static profiles and read out the pressure values for each intersection position. In figure $3(\mathrm{~b})$ and (c), we plot these read-out pressure values using symbols as a function of position for the two different flow rates ; solid (red) lines are the deflection profiles; also plotted $(+)$ are results from simple flow simulations (see below for further discussion). In figure $3(\mathrm{~b})$, a noticeable deviation from a linear pressure distribution is present, as captured by the two dashed line segments with different slopes. The $p(x)$ in figure 3(c) can be approximated well by a linear fit (dashed line) to within our resolution. In the region near the boundaries ( $x=0 \mathrm{~mm}$ and $x=15.7 \mathrm{~mm}$ ), where significant pressure gradients must be present, it is not possible to obtain pressure readings. Thus, the test section is the shaded regions in figure 3(b) and (c) away from the boundaries. We confirm that similar behavior is observed in all measurements on different channels.

Finally, we show that what is found above is indeed the pressure distribution in the channel. First, we turn to the simple flow simulation results (shown by + ) in figure $3(\mathrm{~b})$ and $(\mathrm{c})$. Here, we take a two-dimensional channel with two rigid walls, with the top one having the experimentally-measured profile $\zeta(x)$ and the bottom one being flat. We prescribe the velocity $u$ at the inlet based on the experimental $Q$ value. We then calculate the pressure distribution in the channel with the outlet pressure set to zero (see Appendix A for more details). In figure 3(b), a small nonlinearity qualitatively similar to that observed in the experiment is noticeable. Between the experiment and the simulation, there is a small but constant pressure difference $(\sim 300 \mathrm{~Pa})$, which probably mostly comes form the non-zero outlet pressure in the experiment. In figure $3(\mathrm{c})$, we notice a constant pressure difference $(\sim 500 \mathrm{~Pa})$ between experiment and simulation as well; in addition, there is a larger pressure difference towards the inlet. The excess pressure observed in the experiment is probably the pressure that is needed to keep the deformable wall stretched - as the deformability of the wall is completely ignored in the simulation. The wall is stretched more towards the inlet - hence the larger pressure difference. (We estimate that this tension is not present in the buckled wall of figure 3(b).) Overall, the agreement is quite satisfactory.

We can further validate the extracted pressure drop $\Delta p_{e}$ across the (entire) deformable test section against the analytical approximation in (2). Our method provides $\Delta p_{e}$ directly for each flow rate. We illustrate this in figure $3(\mathrm{~b})$ : we take the high and low pressure values at the beginning and end of the test section, and calculate the difference to find $\Delta p_{e}$, i.e., $\Delta p_{e}=p(x \approx 1.6 \mathrm{~mm})-$ $p(x \approx 13 \mathrm{~mm})$. Against this $\Delta p_{e}$ value, we plot $Q R$, where $R$ is the hydraulic resistance of the channel for only the region where the pressure drop is determined, i.e., the hydraulic resistance of the test section. For the data in figure $3(\mathrm{~b})$, for instance, $R=\int_{x \approx 1.6 \mathrm{~mm}}^{x \approx 13 \mathrm{~mm}} r(x) d x$, where $r(x)$ is the resistance per unit length given in (4). $Q R$ vs. $\Delta p_{e}$ data for each channel and flow rate are shown in figure 4 . The error bars are due to the propagated uncertainties in the measurements of $2 h_{0}+\zeta(x)$.

\section{CONCLUSIONS AND OUTLOOK}

The agreement in figures 3 and 4 provides validation for our method and gives us confidence that we can mea- 

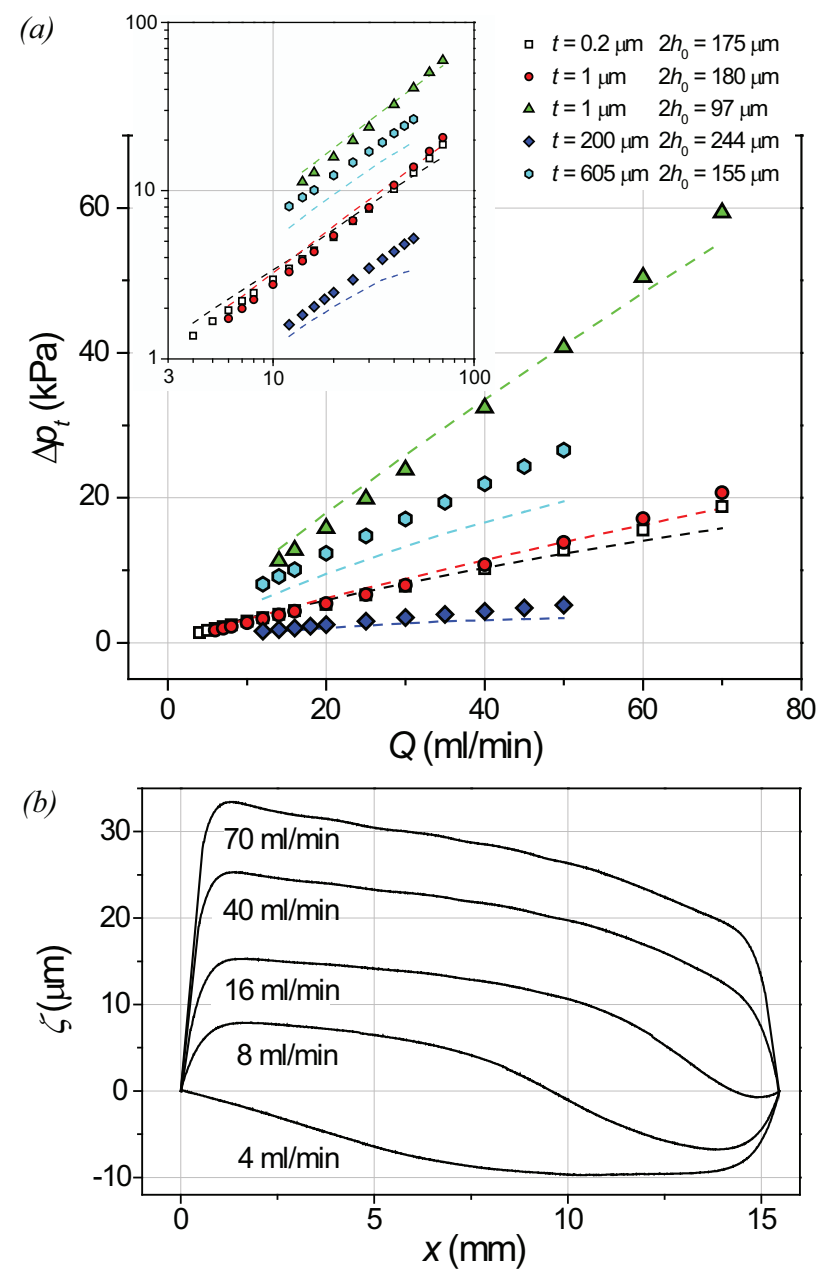

FIG. 2. (a) The pressure drop $\Delta p_{t}$ in the compliant sections of the micro-channels as a function of flow rate $Q$. Error bars are smaller than the symbol sizes. The dotted lines show fits based on the hydraulic resistance of the micro-channel. The inset shows a double logarithmic plot of the same data. (b) The deflection profile $\zeta(x)$ of $\mathrm{S} 1\left(t=200 \mathrm{~nm}, 2 h_{0}=175 \mu \mathrm{m}\right)$ at different $Q$. The profile is no longer uniform [cf. figure 1(d)] because of the position-dependent pressure $p(x)$ in the channel.

sure $p(x)$ in deformable channels accurately. For the proof-of-principle demonstration in this manuscript, we have applied our method to a flow, which can be approximated by the Poiseuille equation, i.e., (2) and (4). However, the method should remain accurate independent of the nature of the flow (e.g., turbulent flows, flows with non-linear $p(x)$ or separated flows) because $p(x)$ simply comes from the wall response - as evidenced by the nonlinear $p(x)$ resolvable in Fig 3(b). It must also be reemphasized that the nature of the wall response is not of consequence as long as the deflection is a continuous function - any function — of pressure, $\zeta=\zeta(p)$. All these suggest that the method can be applied universally as an accurate probe of flows with micron, and even possibly sub-micron, length scales.
Our results may be related to prior studies on collapsible tubes [21, 27]. For our system in two dimensions, in the case of small displacements, $\zeta / 2 h_{0} \ll 1$, one can write a "tube law"

$$
p=p\left(\zeta, \zeta^{\prime \prime}\right) \approx a \zeta+b \zeta^{\prime \prime}
$$

which relates the local the gauge pressure $p$ (the so called transmural pressure) to the channel deflection $\zeta$ and its axial derivative $\zeta^{\prime \prime}=d^{2} \zeta / d x^{2}$. In analogous expressions in the collapsible tube literature, the coefficient $a$ is typically found by considering the changes in the crosssectional area; however, finding $b$, which determines the effect of the axial tension on $p$, is typically not simple and is possible only for certain tube geometries, e.g., for elliptic tubes [28]. Several points are noteworthy about our experiments. First, the axial tension term appears to be unimportant here, i.e., $p=p(\zeta)$. Second, the method remains accurate even when $\zeta \sim 2 h_{0}$. Finally, a method similar to the one described here may be useful for determining $b$ experimentally for different geometries and large deformations.

We mention in passing that the friction drag in a channel with rigid walls separated by a gap of $2 h_{0}$ is larger than that in a deformable channel with the same unperturbed gap. To see this, consider a one-dimensional flow with a flux $q$ per unit width. The stress at the rigid wall is $\tau=-\left.\eta \partial_{y} u\right|_{\text {wall }}=h_{0} \partial_{x} p$. But $q=\frac{2 h_{0}{ }^{3}}{3 \eta} \partial_{x} p$. Therefore, $\tau=\frac{3 \eta^{2} q}{2 h_{0}{ }^{2}}$. Given that $\frac{q}{h_{0}^{2}} \geq \frac{q}{\left(h_{0}+\zeta / 2\right)^{2}}$, drag is reduced. We also note that no evidence of transition to turbulence has been observed in our experiments even at the largest $\mathrm{Re} \approx 1200$.

This noninvasive method can possibly find applications in characterizing physiological flows. In blood flow in arteries [29] and smaller vessels [4], flow-structure interactions are critical in determining functionality [21, 30, 31]. Using our method, for example, one could extract local pressure distribution in an arterial aneurysm, where the arterial wall degrades and eventually ruptures due to the pressure and shear forces during blood flow 32.

The spatial resolution in a $p(x)$ measurement depends upon the resolution in $\zeta$, the noise in the hydrostatic pressure measurement, and the magnitude of the response of the wall. With our current imaging system, we can detect deflections with $\lesssim 20 \mathrm{~nm}$ precision, and the r.m.s. noise in the hydrostatic pressure transducer is $\sim 10 \mathrm{~Pa}$. By collecting the constitutive curves in figure 3(a) at smaller pressure intervals, we estimate that we can measure $p(x)$ with $\sim 10 \mu \mathrm{m}$ resolution in this particular channel. This method can easily be scaled down to provide sub-micron resolution in a nano-fluidic channel by employing a higher numerical aperture objective. It may also be possible to extend the method to study timedependent fluid-structure interactions 33, 34] by collecting surface deformation maps faster [35]. By optimizing the averaging time, one should be able to collect highspeed high-resolution pressure measurements in miniaturized channels. Such advances could open up many 
(a)
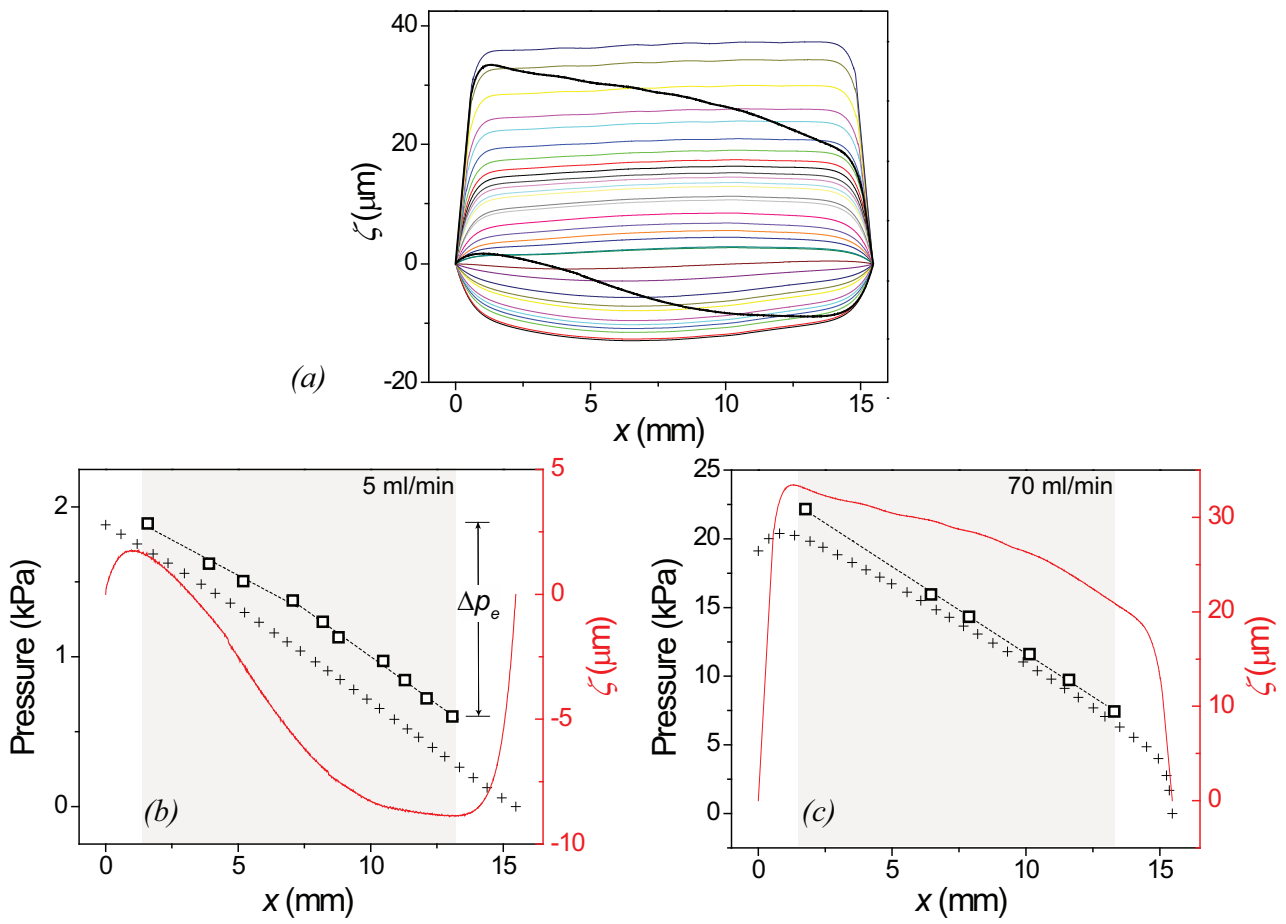

FIG. 3. Determining the pressure gradient inside a microchannel from hydrostatic measurements $\left(\mathrm{S} 1, t=200 \mathrm{~nm}, 2 h_{0}=175 \mu \mathrm{m}\right)$. (a) The channel deflection profiles under two flow rates (upper thick line, $Q=70 \mathrm{ml} / \mathrm{min}$; lower thick line, $Q=5 \mathrm{ml} / \mathrm{min}$ ) are overlaid on top of the deflection profiles taken under different hydrostatic pressures [cf. figure 1(d) and figure 2(b)]. (b) The data points correspond to the pressure distribution $p(x)$ in the test section (shaded region) for a flow rate of $Q=5 \mathrm{ml} / \mathrm{min}$. To obtain $p(x)$, the pressure values at the intersection points in (a) are plotted as a function of $x$. A nonlinearity is noticeable in $p(x)$ at $x \approx 7.5 \mathrm{~mm}$, where the slope of the linear fit changes. (c) Similarly determined $p(x)$ for $Q=70 \mathrm{ml} / \mathrm{min}$. The dashed line is a simple linear fit. The solid (red) lines in (b) and (c) are the deflection profiles of the channel at the given flow rates. The error bars in the data are estimated to be smaller than the symbol size. Also plotted $(+)$ are results from simple flow simulations.

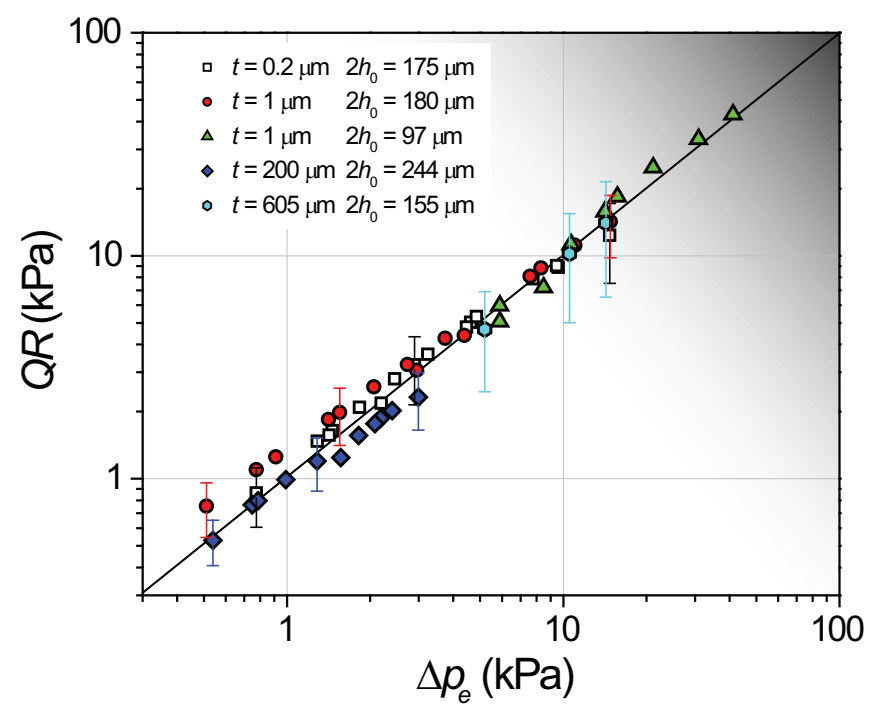

FIG. 4. Calculated pressure drop $Q R$ in the test section as a function of the extracted pressure drop $\Delta p_{e}$. The symbols match with those used in figure 2(a). Representative error bars are due to the uncertainties in $2 h_{0}+\zeta(x)$. The solid line is the $Q R=\Delta p_{e}$ line. other interesting fluid dynamics problems, especially in biological systems.

\section{ACKNOWLEDGEMENTS}

We acknowledge generous support from the US NSF through Grant No. CMMI-0970071. We thank Mr. Le Li for help with the flow simulations.

\section{Appendix A}

Pressure drops in the rigid inlet and outlet regions of the channels are deduced from flow simulations in Comsol Multiphysics using the single-phase 3D steady laminar flow environment. A constant volumetric flow rate is applied at the inlet port, and the pressure at the outlet port is kept at zero. All the channel walls are assigned the no-slip boundary condition. We use quadrilateral mesh elements and increase the mesh density until the results converge. The $2 \mathrm{D}$ simulations shown in figure $3(\mathrm{~b})$ and (c) between the deformed top wall and the flat bottom wall are carried out using the same single-phase steady 
laminar flow environment. The upper compliant wall is replaced with a rigid wall, but the deformed wall shape is preserved by importing the experimental profile into the simulation. At the inlet port, instead of volumetric flow rate, the calculated flow velocity corresponding to the experimental volumetric flow rate is used.
[1] S. P. Sutera and R. Skalak, Annu. Rev. Fluid Mech. 25, 1 (1993).

[2] B. J. McKeon, Springer Handbook of Experimental Fluid Mechanics, edited by C. Tropea, A. L. Yarin, and J. F. Foss (Springer-Verlag, Berlin, 2007).

[3] H. H. Bruun, Hot-wire Anemometry: Principles and Signal Analysis, (Oxford University Press, Oxford, 1995).

[4] A. S. Popel and P. C. Johnson, Ann. Rev. Fluid Mech. 37, 43 (2005).

[5] H. A. Stone, A. D. Stroock, and A. Ajdari, Annu. Rev. Fluid Mech. 36, 381 (2004).

[6] R. B. Schoch, J. Han, P. Renaud, Rev. Mod. Phys. 80, 839 (2008).

[7] C. Lissandrello, V. Yakhot, and K. L. Ekinci, Phys. Rev. Lett. 108, 084501 (2012).

[8] K. L. Ekinci, D. M. Karabacak, and V. Yakhot, Phys. Rev. Lett. 101, 264501 (2008).

[9] K. L. Ekinci, V. Yakhot, S. Rajauria, C. Colosqui and D. M. Karabacak, Lab Chip 10, 3013 (2010).

[10] B. S. Hardy, K. Uechi, J. Zhen, and H. P. Kavehpour, Lab Chip 9, 935 (2009)

[11] M. J. Kohl, S. I. Abdel-Khalik, S. M. Jeter, D.L. Sadowski, Sens. Actuators A Phys. 118, 212 (2005).

[12] A. Orth, E. Schonbrun, and K. B. Crozier, Lab Chip 11, 3810 (2011).

[13] W. Song, and D. Psaltis, Biomicrofluidics 5, 044110 (2011).

[14] N. Srivastava, and M. A. Burns, Lab Chip 7, 633 (2007).

[15] M. Akbarian, M. Faivre, and H.A. Stone, PNAS 103, 538 (2006).

[16] T. Gervais, J. El-Ali, A. Günther, and F.K. Jensen, Lab Chip 6, 500 (2006).

[17] G. M. Whitesides, and A. D. Stroock, Physics Today 54, 42 (2001).

[18] D. P. Holmes, B. Tavakol, G. Froehlicher, and H. A. Stone, Soft Matter, 10.1039/C3SM51002F (2013).
[19] A. E. Hosoi, and L. Mahadevan, Phys. Rev. Lett. 93, 137802 (2004).

[20] M. Shelley, N. Vanderberghe, and J. Zhang, Phys. Rev. Lett. 94, 094302 (2005).

[21] M. Heil, and O. Jensen, IUTAM Symposium on Flow Past Highly Compliant Boundaries and in Collapsible Tubes, edited by P.W. Carpenter, T.J. Pedley (Kluwer Academic, Dordrecht, The Netherlands, 2003).

[22] T. Pedley, and X. Y. Lou, Theor. Comput. Fluid Dyn. 10, 277 (1998).

[23] L. Deck, and P. de Groot, Applied Optics 33, 7334 (1994)

[24] M. K. Small, and W. D. Nix, J. Mater. Res. 7, 1553 (1992).

[25] G.K. Batchelor, An Introduction to Fluid Dynamics (Cambridge University Press, New York, 2000).

[26] H. Bruss, Theoretical Microfluidics (Oxford University Press, New York, 2008)

[27] P. W. Carpenter, and T. J. Pedley (Ed.), IUTAM Symposium on Flow Past Highly Compliant Boundaries and in Collapsible Tubes (Kluwer Academic, Dordrecht, The Netherlands, 2003)

[28] R. J. Whittaker, M. Heil, O. E. Jensen, and S. L. Waters, Q. J. Mech. Appl. Math 63, 465 (2010)

[29] D. N. Ku, Annu. Rev. Fluid Mech. 29, 399 (1997).

[30] J. B. Grotberg, and O. E. Jensen, Annu. Rev. Fluid Mech. 36, 121 (2004).

[31] M. Heil, J.Fluid Mech. 353, 285 (1997).

[32] J. C. Lasheras, Annu. Rev. Fluid Mech. 39, 293 (2007).

[33] C. D. Bertram, and J. Tscherry, J. Fluids Struct. 22, 8, 1029 (2006).

[34] L. Huang, J. Fluids Struct. 15, 7, 1061 (2001).

[35] A. Sampathkumar, K. L. Ekinci, and T. W. Murray, Nano Lett. 11, 1014 (2011). 